\title{
Phenolics profile of a naturally debittering olive in comparison to regular olive varieties ${ }^{\dagger}$
}

\section{A Burcu Aktas, Banu Ozen, ${ }^{*}$ Figen Tokatli and Ilknur Sen}

\begin{abstract}
BACKGROUND: Hurma, an olive variety that grows in a specific area in Turkey, loses its bitterness before harvesting, and therefore does not need further processing steps for the production of table olives. The total phenol content and phenolic profiles of (1) this naturally debittered olive type, Hurma; (2) the same olive variety, but not a naturally debittered type, Erkence; and (3) another variety, Gemlik, which is commonly consumed as table olive, were determined during their maturation period for two harvest years.
\end{abstract}

RESULTS: The total phenol content of Hurma is the lowest compared to the other types regardless of harvest year, which has a significant effect on the phenolic content and composition of individual components for all olive types. All three olive types can be differentiated from each other especially during the late phase of maturation using the phenolics profile in combination with principal component analysis.

CONCLUSION: The natural debittering phenomenon of Hurma olive on the tree involves a decrease in phenol content and a change in phenol composition. The differentiation in phenol composition especially becomes very significant in the late of period of maturation.

(c) 2013 Society of Chemical Industry

Keywords: olive; phenolics; maturation; debittering

\section{INTRODUCTION}

Table olives are defined as the sound fruit of varieties of the cultivated olive trees (Olea europaea L.) that are chosen for their production of olives whose volume, shape, flesh-to-stone ratio, fine flesh taste, firmness and ease of detachment from the stone make them particularly suitable for processing. These olives are treated to remove their bitterness and preserved by natural fermentation; or by heat treatment, with or without the addition of preservatives. Finally, the product is packed with or without a covering liquid. ${ }^{1}$ Olive type, mostly Erkence, grown in nearby area around Karaburun peninsula of Izmir, Turkey, goes through a natural debittering phase on the tree during its ripening. This naturally debittered olive is known by the name of Hurma. At the end of this process, the olive loses its bitter taste while still on the tree and has a dark brownish colour in the inside and a wrinkled outer layer which are its differentiating appearance characteristics from olives that do not undergo this process. Since Hurma olive is already debittered on the tree it does not need to go through further table olive processing steps that other table olives are required to. Therefore, consumers having health problems such as hypertension problem prefer this olive type over table olives that have a higher salt content. A similar type of olive is encountered in Greece and is known by the name of 'thrubolea' or 'Throuba Thassos' ${ }^{2,3}$ Another variety, Dhokar, is also reported by researchers from Tunisia. ${ }^{4,5}$ According to an old study performed in Greece with a similar type of olives, the debittering process is the result of an action of a fungus, Phoma olea, which hydrolyses oleuropein. ${ }^{6}$ For the debittering process on the tree the effect of climate and/or soil was also mentioned as the parameter by the local people since this phenomenon is confined only to certain geographical locations.

Table olives are very good sources of phenolic compounds. Phenolic compounds not only have antimicrobial and antioxidant properties but also contribute to preventing Dacus olea infestation of olive. They also play a role in the formation of the black colour of olives through a browning reaction ${ }^{7}$ and their amounts affect textural and sensorial qualities of olives. ${ }^{8}$ In addition, phenolic compounds of olives, especially oleuropein and hydroxytyrosol, are associated with positive health effects such as the prevention of cardiovascular disease, degenerative disease protection, anti-inflammatory and anti-carcinogenic activities due to their antioxidant characteristics., ${ }^{9,10}$

It was determined that Throuba Thassos had a higher oleuropein content compared to other varieties according to a study performed with nine Greek olive types. ${ }^{3}$ In another study, the phenol profile of the Dhokar variety grown in Tunisia was followed up during its maturation period and was compared with a common variety, Chemlali. ${ }^{4}$ Oleuropein was the main phenolic compound for both varieties at the early stages of ripening; however, the amount of this phenolic compound decreased with maturation and the hydroxytyrosol concentration increased. It was reported

\footnotetext{
* Correspondence to: Banu Ozen, Izmir Institute of Technology, Department of Food Engineering, Urla-Izmir, Turkey.E-mail:banuozen@iyte.edu.tr

† Part of this paper was presented at the EuroFoodChem XVII, 7-10 May 2013.

Izmir Institute of Technology, Department of Food Engineering, Urla-Izmir, Turkey
} 
that the oleuropein concentration decreased to very low levels while it was still in measurable amounts in Chemlali before harvest.

The aim of this study was to determine both total the phenol content and phenolics profiles of the naturally debittering olive type, Hurma, throughout 8 weeks of the maturation period and to compare these data with those obtained for Erkence, the same variety, which does not go through the debittering phase, and for Gemlik, a variety commonly consumed as table olives.

\section{EXPERIMENTAL}

\section{Olive samples}

Three different types of olive were used in the analysis: Gemlik (G), Erkence $(E)$ and Hurma $(H)$ (naturally debittering Erkence) olives. Hurma and Erkence olives were hand-picked from an olive orchard (latitude $38^{\circ} 54^{\prime} 07^{\prime \prime} \mathrm{N}$, longitude $26^{\circ} 57^{\prime} 24^{\prime \prime} \mathrm{E}$ ) which is located in Karaburun Peninsula of Izmir, while the Gemlik type was obtained from another orchard located in Izmir Institute of Technology campus area (latitude $38^{\circ} 19^{\prime} 30.84^{\prime \prime} \mathrm{N}$, longitude $26^{\circ} 37^{\prime} 48.87^{\prime \prime}$ E) which is $30 \mathrm{~km}$ south of first orchard.

For the two harvest years (2011 and 2012) all olives were picked up during 8 weeks of the maturation period from the end of October until the beginning of December. Every week approximately half a kilogram of olives was picked up from the all sides of three trees for each type.

After harvesting, the kernels of olives were separated from the fruit immediately. For the storage, olives were first immersed in liquid nitrogen, then dried with a freeze dryer (Labconco, Kansas City, MO, USA). The maturity index of the olives was determined before lyophilisation process according to a procedure described in the literature. ${ }^{11}$ In order to calculate the maturity index, 100 olives were selected at random, classified into seven groups according to their colour (green, black, reddish brown etc.) and olives in each group were counted. Black olives were cut up to examine the percentage of olive flesh turning to black or purple. The counted olive samples were multiplied with different coefficient numbers for each class and a formula given by Morello et al. ${ }^{11}$ was used to determine the maturity index.

\section{Chemicals}

Reagents used in the chemical analysis were obtained from Riedelde-Haën (Munich, Germany) and Sigma-Aldrich (Munich, Germany) and they were either of high-performance liquid chromatography (HPLC) or analytical grade.

\section{Total phenol content measurement}

The concentrations of total phenolic compounds of olives were determined with the Folin-Ciocalteu assay. ${ }^{12}$ Lyophilised olive pulp $(0.3 \mathrm{~g})$ was weighed and extracted with $5 \mathrm{~mL}$ methanol five times. The methanol in the extract was evaporated at $45^{\circ} \mathrm{C}$ with a rotary evaporator (Laborato 4000; Heidolph, Schwabach, Germany) in $20 \mathrm{~min}$. The remaining extract was dissolved in $5 \mathrm{~mL}$ methanol again. One hundred microlitres of this extract was taken into a glass tube and $2.5 \mathrm{~mL}$ of Folin-Ciocalteu reagent (diluted 10 times with water) was added. Then, $2 \mathrm{~mL}$ of $\mathrm{Na}_{2} \mathrm{CO}_{3}\left(75 \mathrm{~g} \mathrm{~L}^{-1}\right)$ was added within a time interval from 0.5 to $8 \mathrm{~min}$. The sample was incubated at $40^{\circ} \mathrm{C}$ for $15 \mathrm{~min}$ and then cooled to room temperature. The absorbance of the sample was measured at $765 \mathrm{~nm}$ with a UV spectrophotometer (PG Instruments, Leicestershire, UK) and distilled water was used as the blank. Total phenol content of the extracts was calculated using the standard curve and the results were expressed in milligrams of gallic acid equivalent per $100 \mathrm{~g}$ of dry matter (mg GAE $100 \mathrm{~g}^{-1} \mathrm{DW}$ ).

\section{Phenolic compounds profile}

The phenolic extracts were obtained according to a procedure in the literature. ${ }^{13}$ One gram of lyophilised olive pulp was extracted with $15 \mathrm{~mL}$ EtOH-water (80:20) twice. Then, $1 \mathrm{~mL}$ internal standard (150 ppm 4-hydroxyphenyl acetic acid) was added and the mixture was homogenised at $15000 \mathrm{rpm}$ (Heidolph) for $5 \mathrm{~min}$. Then, it was filtered through a vacuum filtration unit using a filter paper with a pore size of $125 \mathrm{~mm}$ (Schleicher and Schuell). The filtered extract was put in a rotary evaporator (Laborato 4000; Heidolph), and the ethanol was evaporated at $40^{\circ} \mathrm{C}$ for $20 \mathrm{~min}$ under vacuum. Remaining traces of ethanol were removed by using a nitrogen flow. The extract was washed twice with $30 \mathrm{~mL}$ hexane in order to remove the oil. Hexane, oil and extract were separated from each other by using a separation funnel. Traces of hexane were removed by using a nitrogen flow. The extract was made up to $25 \mathrm{~mL}$ with water-MeOH (70:30) and filtered by $0.45 \mu \mathrm{m}$ syringe filter into vials. Finally, it was injected into the HPLC equipment. Each sample was extracted and analysed twice with HPLC.

Chromatographic analyses were performed with an Agilent 1200 high-performance liquid chromatograph (Agilent, Santa Clara, CA, USA), equipped with a refractive index detector and a photodiode array detectors, a column oven, and an auto sampler. An ODS column (SGE 8211) with $250 \mathrm{~mm} \times 4 \mathrm{~mm}$ (inside diameter) and $5 \mu \mathrm{m}$ (pore size) dimensions was used to analyse the phenolic compounds. Separation was achieved by gradient elution using an initial composition of $95 \%$ mobile phase $\mathrm{A}(0.1 \%$ acetic acid in water) and $5 \%$ mobile phase $B$ (methanol-acetonitrile-acetic acid (50:50:0.1)). The concentration of $B$ was increased to $55 \%$ in 45 min and maintained there for $10 \mathrm{~min}$. Then, the concentration of $B$ was further increased to $100 \%$ in $5 \mathrm{~min}$ and kept there for $5 \mathrm{~min}$. Finally, $B$ was reduced to $5 \%$ in 5 min and maintained at this concentration

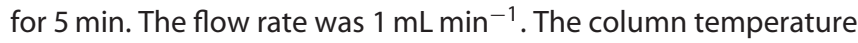
was kept at $30^{\circ} \mathrm{C}$. Chromatograms were obtained at $280 \mathrm{~nm}$ and different phenolic compounds were identified by comparing their retention times with those of commercial standards. Phenolic compounds were quantified by using their respective at least four-point calibration curves and expressed as $\mathrm{mg} \mathrm{kg}^{-1}$ dry weight.

Phenolic compounds used as standards in the analysis were oleuropein, oleuropein aglycone, tyrosol, rutin, hydroxytyrosol, quercetin-3-glucoside, quercetin, luteolin, luteolin-7-glucoside, verbascoside, vanillin, vanillic acid, ferulic acid, o-coumaric acid, p-coumaric acid, syringic acid, caffeic acid, apigenin and apigenin7-glucoside.

\section{Statistical analysis}

The multivariate data matrix consists of observations represented by samples from three different olive types for two harvest years and variables represented by the total phenol content (TPC), together with individual phenolics composition. The same analysis was also performed for each harvest year by separating the data into two to observe the differences between olive types more clearly. Data were autoscaled before multivariate analysis. The data matrix was analysed by principal component analysis (PCA). The multivariate analyses were performed by SIMCA-P v.11.5 (Umetrics, Umea, Sweden). Results of PCA are visualised by scores and loading plots. Score plots were constructed to observe principal groupings among observations. Loadings indicate the importance of each variable for the model and loading plots are used to interpret the relations among variables and clusters observed in the score plots. 
Table 1. Maturity index, total phenol content $\left(\mathrm{mg}_{\left.100 \mathrm{~g}^{-1}\right) \text { and concentration of individual phenolic compounds (mg kg }}{ }^{-1}\right)$ of Hurma, Erkence and Gemlik olive types during 8 weeks of maturation period for two harvest years

\begin{tabular}{|c|c|c|c|c|c|c|c|c|c|c|c|c|c|c|c|c|c|}
\hline Olive type & $\mathrm{MI}$ & TPC & OLE & HYT & TY & API & VER & RTN & L-7-Glu & LTLN & QUE & o-cou & $p$-cou & $\mathrm{FA}$ & VA & CA & VN \\
\hline \multicolumn{18}{|l|}{$2011 / 2012$} \\
\hline $\mathrm{H} 11$ & 3.90 & 560.40 & 527.63 & 2013.44 & 17.49 & 10264.62 & 517.20 & 9.57 & 709.98 & 36.37 & 18.60 & 61.42 & 0.59 & ND & 2.16 & 2.41 & 0.79 \\
\hline $\mathrm{H} 12$ & 4.10 & 649.64 & 786.15 & 2722.70 & 36.91 & 1022.18 & 66.37 & 12.99 & 84.07 & 34.20 & 23.20 & 118.91 & 0.70 & 0.57 & 3.01 & 2.37 & 1.62 \\
\hline $\mathrm{H} 13$ & 5.34 & 523.26 & 1166.89 & 1424.46 & 18.68 & 297.04 & 54.05 & 10.04 & 128.86 & ND & 35.13 & 122.78 & 1.05 & ND & 2.07 & 3.88 & ND \\
\hline $\mathrm{H} 14$ & 4.95 & 412.68 & 87.70 & 3357.91 & 11.57 & 1727.42 & 81.92 & ND & 123.80 & 4.99 & ND & 126.62 & ND & 0.81 & 3.04 & ND & 1.89 \\
\hline $\mathrm{H} 15$ & 3.74 & 337.68 & ND & 827.59 & ND & 377.30 & 57.20 & ND & 7.49 & ND & ND & ND & 5.09 & ND & 3.49 & 4.44 & ND \\
\hline $\mathrm{H} 16$ & 4.37 & 533.97 & 103.71 & 1602.36 & 52.51 & 15502.65 & 519.73 & 9.56 & 189.07 & 72.98 & 35.96 & 16.09 & 1.99 & 231.73 & 5.75 & 1.66 & 4.57 \\
\hline $\mathrm{H} 17$ & 3.99 & 579.68 & 753.08 & 4104.61 & 73.11 & 8003.25 & 1995.76 & 21.22 & 334.76 & ND & 50.41 & 23.34 & 3.86 & 282.28 & 10.73 & 2.14 & 4.27 \\
\hline $\mathrm{H} 18$ & 3.94 & 644.63 & 241.91 & 3239.12 & 32.54 & 5561.71 & 391.09 & 24.88 & 532.48 & ND & 47.51 & 47.82 & 3.08 & ND & 9.41 & ND & 2.29 \\
\hline E11 & 0.50 & 518.43 & 1388.21 & 1001.16 & 14.94 & 3993.87 & 258.76 & 12.23 & 167.93 & 69.51 & 26.18 & 103.98 & ND & ND & ND & 2.98 & ND \\
\hline E12 & 1.27 & 518.02 & 1265.98 & 1627.43 & 24.19 & 1270.94 & 31.02 & 13.76 & 141.60 & 98.42 & 27.66 & 170.52 & 1.46 & ND & 4.94 & 3.03 & ND \\
\hline E13 & 2.25 & 526.22 & 625.71 & 2222.26 & 30.42 & 1391.48 & 39.24 & 59.50 & 63.43 & ND & 33.73 & 12.51 & ND & ND & 11.52 & 2.86 & ND \\
\hline E14 & 1.65 & 520.95 & 137.99 & 497.87 & 0.94 & 990.58 & 14.52 & ND & 22.13 & 109.30 & 17.56 & ND & ND & ND & 3.43 & 1.48 & ND \\
\hline E15 & 1.69 & 504.69 & 470.91 & 471.66 & 11.03 & 1673.22 & 40.33 & 13.13 & 56.83 & 95.03 & 12.55 & 30.49 & 2.91 & ND & 3.64 & 2.12 & ND \\
\hline E16 & 3.65 & 900.69 & 329.34 & 2011.45 & 29.03 & 15454.53 & 312.88 & 15.00 & 1387.23 & 168.43 & 37.88 & 74.07 & 1.90 & ND & ND & 1.42 & 2.07 \\
\hline E17 & 3.56 & 691.32 & 608.61 & 1281.41 & 19.14 & 9804.94 & 566.54 & 29.24 & 1833.22 & 351.19 & 95.64 & 143.83 & 2.31 & ND & 2.61 & 2.70 & 2.18 \\
\hline E18 & 3.67 & 1230.44 & 705.14 & 876.30 & 12.36 & 4443.14 & 424.94 & 29.53 & 2207.42 & 252.30 & 63.11 & 203.62 & 2.85 & ND & 1.50 & 2.12 & 1.75 \\
\hline G12 & 1.10 & 544.61 & 4786.76 & 3070.73 & ND & 4788.42 & 594.31 & ND & 78.70 & ND & ND & 135.08 & ND & ND & ND & ND & ND \\
\hline G13 & 1.38 & 452.33 & 2057.32 & 5399.42 & ND & 2030.06 & 700.34 & 56.85 & 239.39 & 154.25 & ND & 111.69 & ND & 4.33 & ND & ND & ND \\
\hline G14 & 2.41 & 637.10 & 294.53 & 6596.22 & ND & 1835.13 & 66.63 & 24.78 & 133.99 & 171.68 & ND & 107.23 & ND & 5.64 & 7.96 & ND & 11.51 \\
\hline G15 & 2.10 & 416.78 & ND & 2277.51 & ND & 2704.82 & 35.45 & 0.17 & 85.44 & 167.16 & ND & 31.68 & 1.19 & 0.88 & 5.15 & ND & ND \\
\hline G16 & 3.65 & 806.88 & 683.95 & 3803.16 & 17.36 & 24689.41 & 473.78 & 22.11 & 619.82 & 298.15 & 152.09 & 132.76 & 4.39 & ND & 3.67 & ND & 2.13 \\
\hline G17 & 4.82 & 524.48 & 237.30 & 3704.21 & 7.00 & 22139.33 & 689.25 & 19.60 & 957.06 & 128.93 & 30.91 & 112.55 & 3.24 & ND & 2.38 & 1.54 & 1.08 \\
\hline G18 & 5.88 & 701.84 & 307.42 & 8183.35 & 17.09 & 31838.78 & 1942.05 & 74.96 & 1081.14 & ND & ND & 116.47 & 7.24 & ND & ND & 9.54 & ND \\
\hline \multicolumn{18}{|l|}{$2012 / 2013$} \\
\hline $\mathrm{H} 21$ & 5.43 & 208.36 & 780.77 & 61.83 & ND & 1251.18 & 76.71 & 33.65 & 22.29 & ND & ND & 8.27 & ND & 12.02 & 11.03 & ND & ND \\
\hline $\mathrm{H} 22$ & 4.56 & 344.34 & 190.95 & 22.56 & ND & 539.02 & 10.82 & 28.91 & 7.88 & ND & ND & 3.13 & ND & ND & 5.04 & ND & 0.43 \\
\hline $\mathrm{H} 23$ & 5.92 & 73.89 & 60.28 & 33.39 & ND & 378.72 & ND & ND & 9.57 & ND & ND & 0.84 & 0.33 & 5.11 & ND & ND & ND \\
\hline $\mathrm{H} 24$ & 5.60 & 29.21 & 291.10 & 105.61 & 7.55 & 906.07 & 39.90 & 7.78 & 39.28 & ND & ND & 1.09 & 1.03 & 2.96 & ND & 4.41 & ND \\
\hline $\mathrm{H} 25$ & 6.18 & 245.56 & ND & 29.50 & ND & 552.43 & ND & 3.53 & 9.07 & ND & ND & ND & ND & ND & ND & 0.03 & ND \\
\hline $\mathrm{H} 26$ & 5.42 & 152.11 & ND & ND & ND & ND & ND & ND & ND & ND & ND & ND & ND & ND & ND & ND & ND \\
\hline $\mathrm{H} 27$ & 6.37 & 160.95 & 145.35 & 75.34 & 5.54 & 585.27 & 17.17 & 8.57 & 65.29 & ND & ND & 0.76 & 0.21 & 6.15 & 6.50 & 1.16 & ND \\
\hline $\mathrm{H} 28$ & 6.00 & 159.09 & 17.91 & 7.18 & ND & 714.23 & ND & ND & ND & ND & ND & ND & ND & ND & 3.52 & ND & ND \\
\hline E21 & 2.53 & 335.88 & 1490.37 & 101.52 & 10.95 & 929.83 & 29.92 & 61.84 & 21.73 & 1.03 & ND & 9.46 & 0.49 & 4.64 & 9.04 & ND & 1.15 \\
\hline E22 & 2.16 & 359.17 & 505.48 & 126.92 & 13.19 & 1229.20 & 34.77 & 21.19 & 46.06 & 0.95 & ND & 6.56 & 0.27 & 4.18 & 3.51 & ND & ND \\
\hline E23 & 2.34 & 535.66 & 431.90 & 116.45 & 13.18 & 1641.20 & 16.76 & 15.15 & 54.24 & 2.76 & ND & 0.43 & 2.34 & 5.04 & 2.93 & 5.02 & ND \\
\hline E24 & 2.53 & 518.32 & 307.20 & 123.31 & 10.83 & 1412.50 & 43.73 & 19.96 & 34.27 & 1.20 & ND & ND & 7.85 & 6.66 & 3.52 & 2.64 & 0.43 \\
\hline E25 & 3.17 & 519.82 & 126.38 & 24.11 & 1.79 & 946.92 & 19.16 & 11.86 & 12.45 & 0.64 & ND & 0.49 & 2.91 & 1.71 & 2.00 & 1.85 & ND \\
\hline E26 & 3.18 & 347.00 & 334.58 & 78.59 & 24.77 & 1222.10 & 75.17 & 17.16 & 25.99 & 13.33 & ND & 1.74 & 1.61 & 11.01 & 78.59 & 2.39 & 1.38 \\
\hline E27 & 3.49 & 664.81 & 139.11 & 79.40 & 5.13 & 920.20 & 11.08 & 8.12 & 39.07 & ND & ND & 5.98 & 3.77 & 2.00 & 2.05 & 1.01 & 0.16 \\
\hline E28 & 5.94 & 514.07 & 58.67 & 97.33 & 28.03 & 1211.10 & 80.42 & 30.29 & 21.33 & 56.24 & ND & 8.02 & 0.37 & 8.38 & 2.43 & 1.89 & ND \\
\hline G21 & 2.42 & 343.29 & 242.32 & 118.06 & 7.54 & 634.37 & 88.58 & 34.77 & 13.56 & 39.23 & ND & 6.55 & 0.46 & 14.73 & 3.96 & ND & 0.41 \\
\hline G22 & 2.19 & 411.37 & 751.48 & 484.79 & 10.07 & 1615.70 & 160.85 & 22.33 & 90.51 & ND & ND & 2.66 & 0.93 & 33.11 & 1.96 & ND & ND \\
\hline G23 & 3.32 & 244.92 & 166.65 & 427.43 & ND & 981.71 & 47.10 & 12.75 & 37.64 & 1.34 & ND & 0.92 & 0.38 & 8.07 & 2.12 & 2.12 & ND \\
\hline G24 & 3.09 & 229.63 & 280.47 & 616.73 & 5.66 & 1420.90 & 194.56 & 24.63 & 28.68 & ND & ND & 1.84 & 0.69 & 10.06 & 2.39 & ND & 0.08 \\
\hline$G 25$ & 3.92 & 242.80 & 456.82 & 346.14 & 8.42 & 888.65 & 110.62 & 15.09 & 41.80 & ND & ND & ND & 0.67 & 15.54 & 4.31 & ND & 0.61 \\
\hline G26 & 4.60 & 103.19 & 163.24 & 374.06 & 2.66 & 694.54 & 37.17 & 6.51 & 22.32 & ND & ND & 0.72 & 0.20 & 15.94 & 8.18 & ND & ND \\
\hline G27 & 4.26 & 452.36 & 385.87 & 424.92 & 5.66 & 838.93 & 105.00 & 10.79 & 49.94 & ND & ND & 3.91 & 0.34 & 8.13 & 3.04 & ND & 0.53 \\
\hline G28 & 3.84 & 228.39 & 290.94 & 288.62 & ND & 701.73 & 37.60 & 4.14 & 8.15 & ND & ND & ND & 0.50 & 4.14 & ND & ND & ND \\
\hline $\begin{array}{l}\text { Olive typ } \\
\text { MI, matu } \\
\text { OLE, ole } \\
\text { o-cou, o } \\
\text { Concent }\end{array}$ & n & the ave & f & - & nts & 等 & 年 & 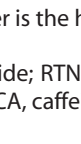 & 年 & . & & & & & & & de; \\
\hline
\end{tabular}


(a)

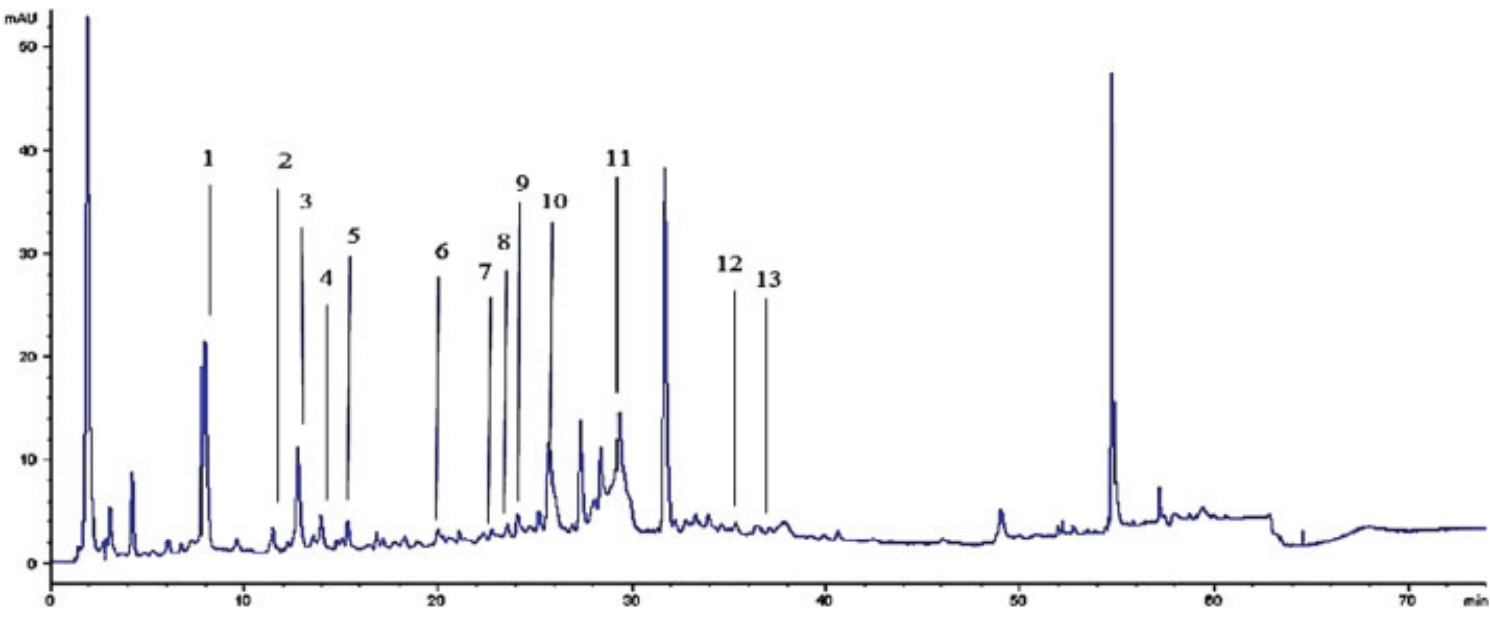

(b)

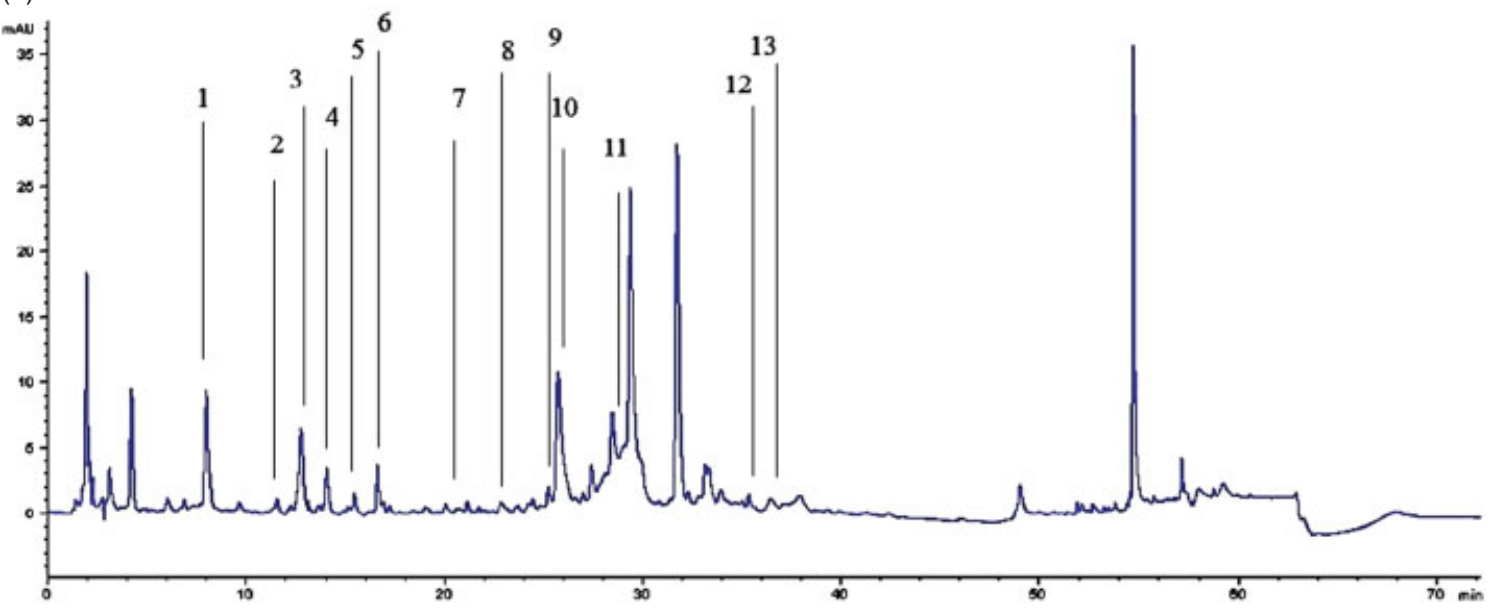

(c)

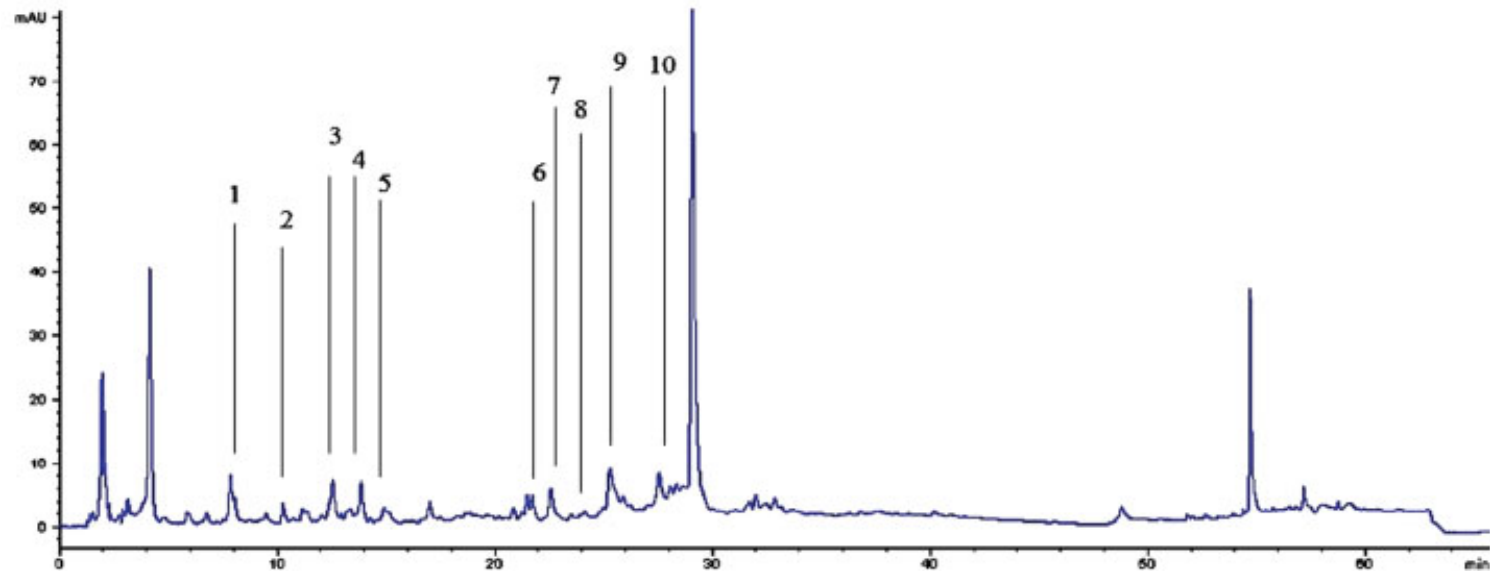

Figure 1. HPLC phenol profiles of (a) Hurma olives: peak 1, hydroxytyrosol; 2, tyrosol; 3, p-hydroxy acetic acid (internal standard); 4, apigenin; 5, vanillic acid; 6, $p$-coumaric acid; 7, verbascoside; 8, luteolin-7-glucoside; 9, rutin; 10, o-coumaric acid; 11, oleuropein; 12, quercetin; 13, luteolin; (b) Erkence olives peak 1, hydroxytyrosol; 2, tyrosol; 3, p-hydroxy acetic acid (internal standard); 4, apigenin; 5 , vanillic acid; 6, caffeic acid; 7, p-coumaric acid; 8, verbascoside; 9, rutin; 10, o-coumaric acid; 11, oleuropein; 12, quercetin; 13, luteolin; and (c) Gemlik olives: peak 1, hydroxytyrosol; 2, tyrosol; 3, $p$-hydroxy acetic acid (internal standard); 4, apigenin; 5, vanillic acid; 6, ferulic acid; 7, verbascoside; 8, rutin; 9, o-coumaric acid; 10, oleuropein). All olives were harvested in the first week of the 2011-2012 harvest year. 

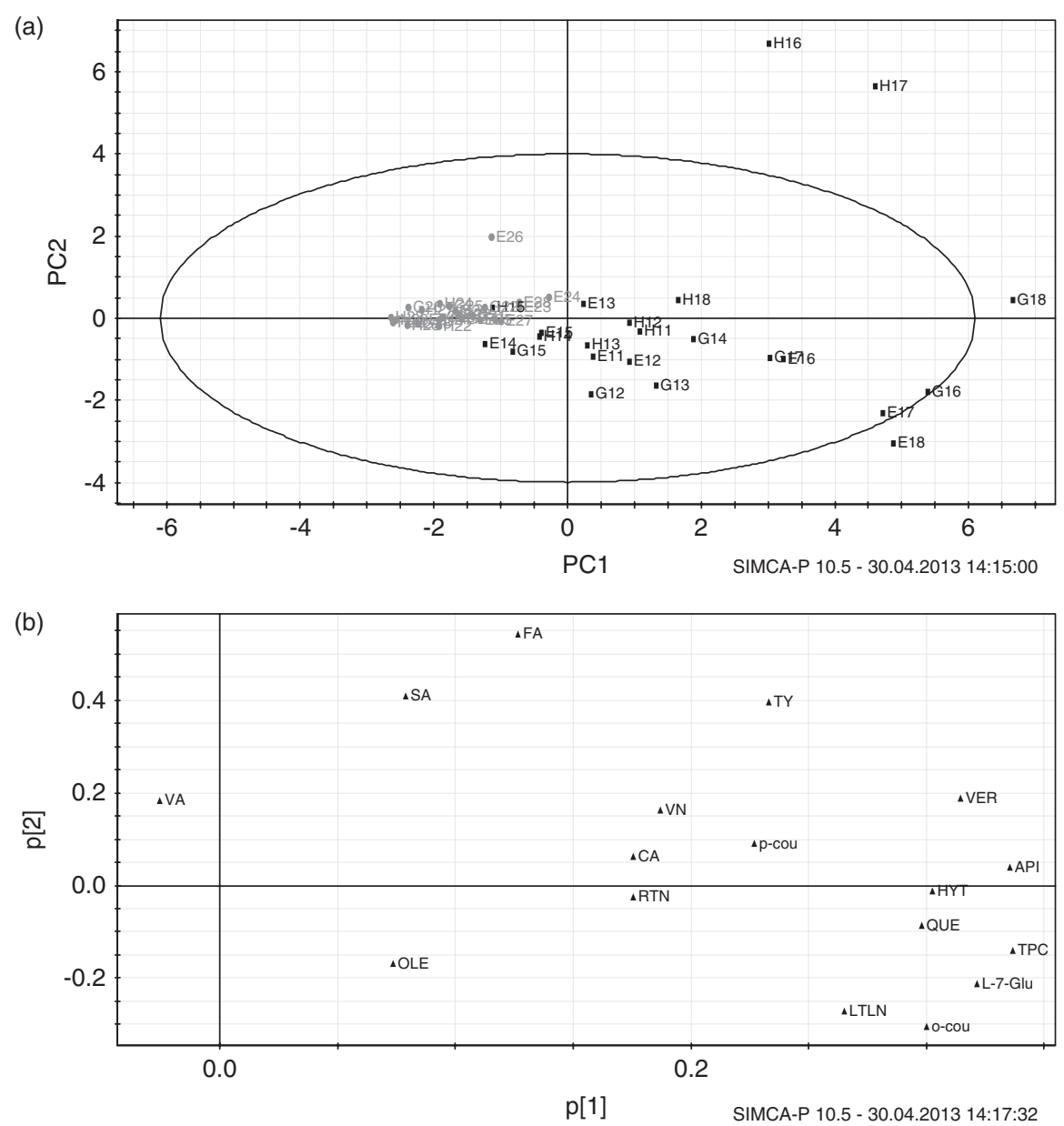

Figure 2. (a) Score plot and (b) loading plot obtained with principal component analysis for total phenol and phenolic profiles of Hurma, Erkence and Gemlik olives during 8 weeks of maturation for the two harvest years ( $\mathrm{H}$, Hurma; E, Erkence; $\mathrm{G}$, Gemlik; the first number after the letter is the harvest year and second number is the harvest week).

\section{RESULTS AND DISCUSSION}

Both in the 2011-2012 and 2012-2013 harvest years olive samples were obtained from the same trees in the same locations from the end of October until the beginning of December. This time corresponds to the season when the appearance of Hurma olives allowed us to differentiate them from regular Erkence olives which did not go through debittering. The maturity index of Hurma ranged from 3.14 to 5.34 in the first season while it was between 4.56 and 6.37 in the next year. Erkence had a range of maturity index of $0.5-3.67$ in 2011-2012 while the range was 2.16-5.94 in 2012-2013. The maturity index of Gemlik varied between 1.1 and 5.88 in the first harvest year and between 2.19 and 4.16 in the second year. The maturity index shows the ripening degree of the fruit and, overall, the maturity index increased during the sampling weeks. Olive samples were collected from the all sides of the same designated trees throughout the sampling period; however, not all olives ripen at the same time. Depending on the position of the trees some sides ripen earlier than others. Therefore, the maturity index increased and decreased in some weeks.

\section{Total phenol content}

Values for the TPC of all three types of olive are listed in Table 1. The TPC of the Erkence variety changed between 504.7 and $1230.4 \mathrm{mg}$ GAE $100 \mathrm{~g}^{-1}$ in 2011-2012, and between 335.9 and $664.8 \mathrm{mg}$
GAE $100 \mathrm{~g}^{-1}$ in the 2012-2013 harvest years, respectively. Hurma had between 337.7 and $649.6 \mathrm{mg} \mathrm{GAE} 100 \mathrm{~g}^{-1}$ TPC in the first harvest year, and between 29.2 and $344.3 \mathrm{mg} \mathrm{GAE} 100 \mathrm{~g}^{-1} \mathrm{TPC}$ in the second harvest year; while the TPC of Gemlik varied between 416.8 and $806.9 \mathrm{mg} \mathrm{GAE} 100 \mathrm{~g} \mathrm{~g}^{-1}$ in the first, and between 103.2 and $452.4 \mathrm{mg} \mathrm{GAE} 100 \mathrm{~g}^{-1}$ in the second year. In general, Erkence had the highest TPC in both harvest years while Hurma, although from the same variety, had a lower phenol content. This difference between these two types of olive might be the result of the debittering stage which Hurma goes through. Dhokar, the Tunisian sweet olive variety, also had a lower TPC (508-768 mg GAE $100 \mathrm{~g}^{-1} \mathrm{DW}$ ) when compared with another olive variety, Chemlali (698-1300 mg GAE $100 \mathrm{~g}^{-1}$ DW) and researchers also attributed the lower TPC of this variety to its sweet character. ${ }^{4}$

During the ripening period the TPC of all olive types increase and decrease. Mostly, there is a decreasing trend after the second week until the firth week, and an increase in TPC follows this decreasing phase. This trend of increase and decrease was also observed in other studies. ${ }^{14}$

In the second harvest year (2012-2013) all three olive types have a lower TPC compared to the first harvest year (2011-2012). A change in the phenol content depending on the harvest year is expected and is well-documented for olive oil in the literature. ${ }^{15,16}$ However, the TPC for different types has similar trend in both years, Erkence having the highest and Hurma having the lowest average 

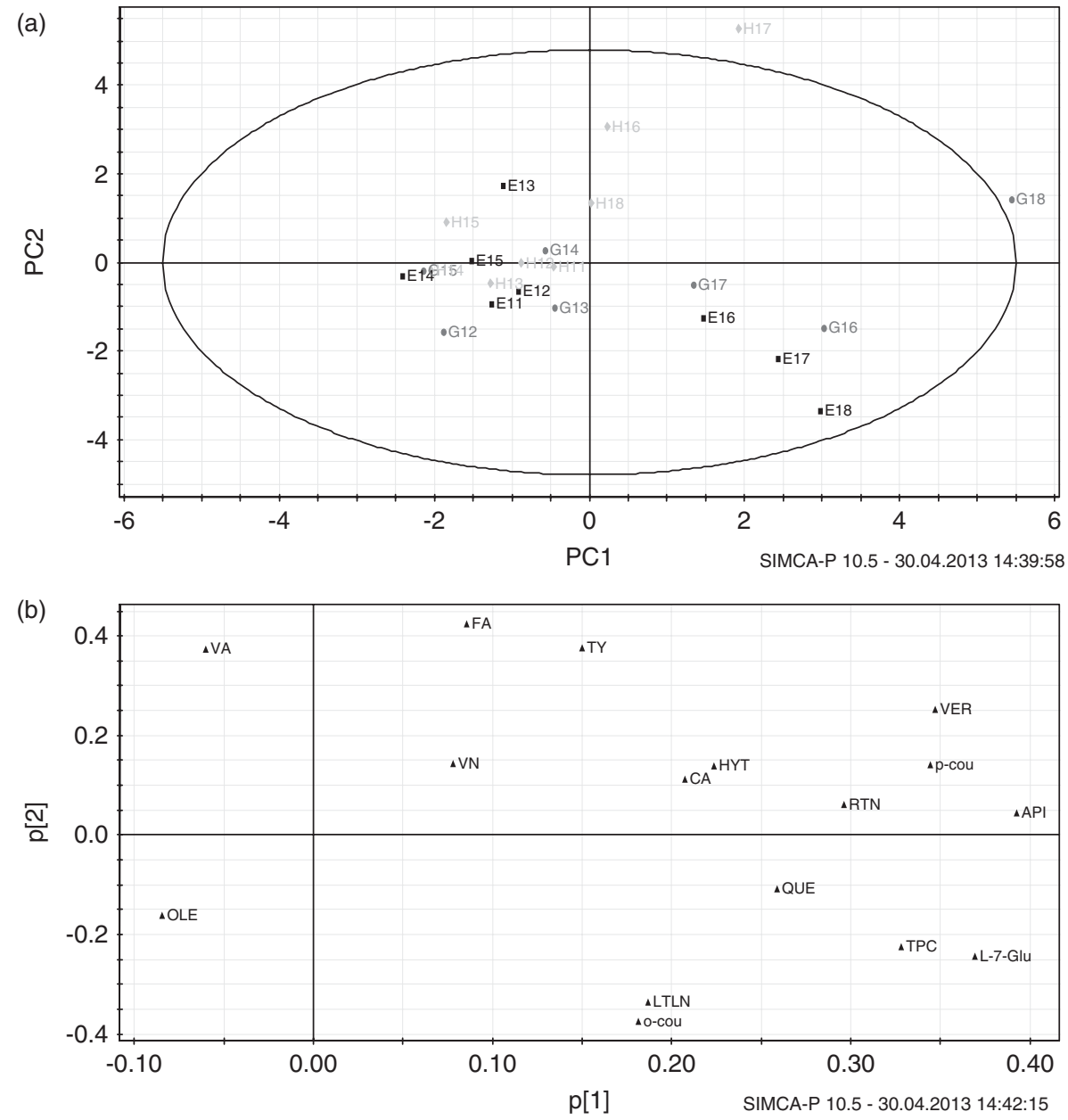

Figure 3. (a) Score plot and (b) loading plot obtained with principal component analysis for total phenol and phenolic profiles of Hurma, Erkence and Gemlik olives during 8 weeks of maturation for the 2011-2012 harvest year ( $H$, Hurma; E, Erkence; $G$, Gemlik; the first number after the letter is the harvest year and second number is the harvest week).

TPC content. ANOVA was used to show the significant differences among TPC values. The $P$ values were found to be less than $10^{-3}$.

\section{Phenolic profiles}

Chromatograms of the phenolic profiles of Erkence, Hurma and Gemlik olive types are provided in Fig. 1, and Table 1 shows the concentration of individual phenolics. As can be seen from the table quercetin-3-glucoside is the only phenol that was detected in the first harvest year in all olive types but not in the second year. In addition, although luteolin was observed in the first year in both Hurma and Gemlik samples, this phenol was not detected in Hurma during the second year, and it was only detected in Gemlik during the first three weeks of the second harvest year. To see the differences between varieties, harvest time and harvest year a multivariate classification technique, PCA, was applied to the data and both TPC and individual phenols were used in the data matrix. Although $R^{2}$ values of the models obtained are not very high, PCA plots are still helpful in visualising the differences regarding the olive type, harvest season and year. Without this multivariate analysis it would be difficult to draw conclusions considering all phenolic compounds and TPC at the same time.

For the whole data, a model with two principal components and $R^{2}$ of 0.48 was obtained and a score plot for this model showing the classification of olive types is provided in Fig. 2a. According to this plot, a differentiation could be observed between the first and the second harvest year olives (except for Hurma during the first season at 5 th weeks of maturation). The first 5 -week samples from all types from the 2011-2012 harvest year are more closely positioned to the 2012-2013 harvest year samples. The later weeks (sixth, seventh and eighth weeks) of the first harvest year are totally separated from the remainder of the samples and are on the right side of the plot. Overall, all phenolic compounds except vanillic acid exist in higher amounts for the samples harvested in the 2011-2012 season. Therefore, they are located on the right side of the loading plot (Fig. 2b) like the first-year olives since they are the differentiating parameters for the first year olives.

To better understand the differences between each type of olives, PCA was run separately for each harvest year and score and loading plots are shown in Fig. 3. PCA constructed for the first harvest year consists of two principal components with an $R^{2}$ of 0.46. The score plot (Fig. 3a) shows also that there is not much differentiation with regard to olive type in the first 5 weeks of sampling. There is a clear separation between olives from the first 5 weeks and the olives harvested in the last 3 weeks. Actually, those last 3 weeks, in general, correspond to the time when most of the harvesting is done locally. According to the loading plot (Fig. 3b) oleuropein and vanillic acid are the phenols that differentiate the early harvest period from the rest. Actually, oleuropein and vanillic 

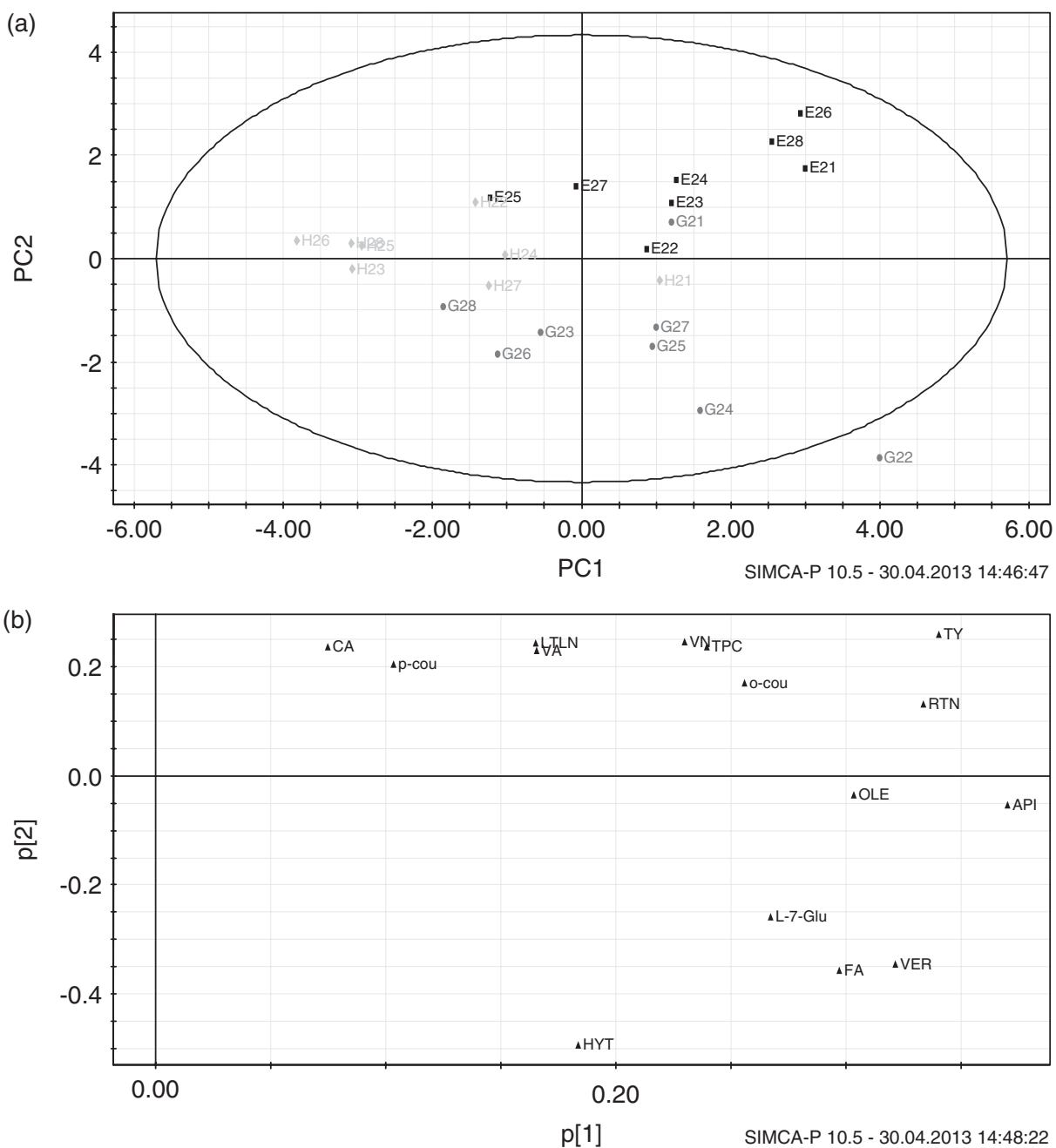

Figure 4. (a) Score plot and (b) loading plot obtained with principal component analysis for total phenol and phenolic profiles of Hurma, Erkence and Gemlik olives during 8 weeks of maturation for the 2012-2013 harvest year ( $H$, Hurma; E, Erkence; $G$, Gemlik; the first number after the letter is the harvest year and second number is the harvest week).

acid contents of early samples are higher compared to the later period (Table 1). When the late period is considered, however, different olive types, especially Erkence and Hurma, could be clearly separated from each other since Erkence is located on the lower right quartile and Hurma on the upper right quartile of the score plot (Fig. 3a). The Gemlik variety in the last 3 weeks of harvest showed a high hydroxytrosol (3704.21-8183.35 $\mathrm{mg} \mathrm{kg}^{-1}$ ) and apigenin (22 139.33-31838.78 $\mathrm{mg} \mathrm{kg}^{-1}$ ) content compared to others. Generally, the oleuropein content of Erkence $\left(137.99-1388.21 \mathrm{mg} \mathrm{kg}^{-1}\right)$ is higher compared to Hurma $\left(0-1166.89 \mathrm{mg} \mathrm{kg}^{-1}\right)$ throughout the sampling period. According to the loading plot (Fig. 3b), luteolin, o-coumaric acid, luteolin7-glucoside and TPC are the differentiating parameters for Erkence. In fact, Erkence has the highest TPC (504.69-1230.44 mg $100 \mathrm{~g}^{-1}$ ) especially in the late period of harvesting while luteolin $\left(168.43-351.19 \mathrm{mg} \mathrm{kg}^{-1}\right)$ exists in high amounts in the final 3 weeks. Vanillin, ferulic acid and tyrosol content of Hurma olives in the last 3 weeks of harvesting are the parameters that separate out this olive from the rest (Fig. 3b).

The PCA model for the second harvest year has an $R^{2}$ of 0.63 and three principal components. PCA in this case provided better classification for different olive types although some samples are not located in their class (Fig. 4a and b). First-week Hurma (H21) and Gemlik (G21) and second-week Erkence (E22) samples are close to each other in the score plot; therefore, separation in the early harvest period is not as clear as observed in the first harvest year. Other than fifth week Erkence (E25), Erkence and Hurma are separated from each other quite well indicating that phenolic compounds are very much affected due to debittering phase during maturation. According to the loading plot (Fig. 4b), hydroxytyrosol, ferulic acid, verbascoside and luteolin-7-glucoside are the phenolics which provide separation of Gemlik type compared to others. Oleuropein, apigenin, rutin and o-coumaric acid are also important phenolics in Gemlik differentiation but they also play a role in Erkence classification since they are located close to horizontal axis. Caffeic acid, p-coumaric acid, vanillin, tyrosol and TPC are the differentiating parameters for Erkence according to the loading plot and this type contains these phenols in higher amounts and its TPC is the highest compared to others as in the first year. Caffeic acid is mostly present in Erkence for the second harvest year. Tyrosol was in significant amounts throughout ripening for Erkence while Hurma did not contain much of this phenolic as opposed to first year. Hurma type has always lower content of every phenolic compounds and especially its oleuropein concentration is very low after 4 weeks in the second harvest year. As reported in the literature, ${ }^{17}$ 
an overall decreasing trend for oleuropein was observed although there are increases and decreases depending on the harvest time. This type of trend during maturation was also observed by other researchers. ${ }^{18}$ Increasing and decreasing trends were observed for almost all phenols and could be explained by the conclusion provided by Menz and Vriesekoop, ${ }^{19}$ that is the continuous synthesis of phenolic compounds during maturation. Since phenolic compounds are secondary metabolites of plants and part of their defence mechanisms they could be synthesised when they are needed depending on the environmental conditions or the other factors. Although a decreasing trend is not that clear for Hurma in the first year it could still be concluded that the amount of oleuropein decreases with increasing maturity index for this olive type also. According to a study performed with sweet Dhokar and regular Chemlali olives there is a clear decrease in oleuropein content and increase in hydroxytyrosol contents of both olives with ripening. While the oleuropein content was reduced to almost zero for Dhokar with time it remained at a certain level for Chemlali. Although the same observation applies for oleuropein in our case, a decreasing trend for hydroxytyrosol is not seen for Hurma as well as other types of olives. Studies on naturally debittered olive varieties were concentrated on the oleuropein content of these olives since oleuropein is the phenolic compound that gives the bitter taste of the olives. However, as observed in this study, not only oleuropein but almost all phenolics are affected by this debittering process. This observation is also confirmed by the lower TPC of the Hurma variety.

\section{CONCLUSION}

Erkence, Hurma and Gemlik olives can be differentiated from each other using their phenolics profiles. Since Hurma is an Erkence type olive which debitters on the tree the separation between Erkence and Hurma shows that natural debittering is related to changes in phenolic composition and this phenomenon results in a reduction in phenolic composition of Hurma. As hypothesised by other researchers these changes in phenolic composition could be related to the activities of $\beta$-glucosidase and esterase enzymes ${ }^{4}$. In addition, the phenolic profiles of olive types investigated in this study depend on harvest year. Therefore, more data on phenolics content obtained at multiple harvest years will be helpful to gain insight into the natural debittering phenomenon of olives. In addition, a comparison between Hurma olives and regular olives after their processing will provide more understanding regarding the health advantages and/or disadvantages of this special type of olive compared with regular olive varieties.

\section{ACKNOWLEDGEMENTS}

This research was supported by a grant provided by the Scientific and Technological Research Council of Turkey (Project No: TOVAG1100780). We would like to thank the Biotechnology and
Bioengineering Research Center of Izmir Institute of Technology for HPLC analysis and Eglenhoca village for providing olive samples.

\section{REFERENCES}

1 International Olive Oil Council (IOOC), Trade standard applying to table olives, IOOC/COI/OT/NC no. 1, December 2004.

2 Panagou EZ, Greek dry-salted olives: Monitoring the dry salting process and subsequent physico-chemical and microbiological profile during storage under different packing conditions at 4 and $20^{\circ}$ C. LWT - Food Sci Technol 39:322-329 (2006).

3 Zoidou E, Melliou E, Gikas E, Tsarbopoulos A, Magiatis P and Skaltsounis AL, Identification of Throuba Thassos, a traditional Greek table olive variety, as a nutritional source of oleuropein. J Agric Food Chem 58:46-50 (2010).

4 Jemai H, Bouaziz M and Sayadi S, Phenolic composition, sugar contents and antioxidant activity of Tunisian sweet olive cultivar with regard to fruit ripening. J Agric Food Chem 57:2961-2968 (2009).

5 Rigane G, Salem RR, Sayadi S and Bouaziz M, Phenolic composition, isolation, and structure of a new deoxyloganic acid derivative from Dhokar and Gemri-Dhokar olive cultivars. J Food Sci 76:C965-C973 (2011).

6 Kalogereas SA, Table Olives. Hermes Publications, Athens (1932).

7 Ryan D, Robards K and Lavee S, Changes in phenolic content of olive during maturation. Int J Food Sci Technol 34:265-274 (2001).

8 Bianco A and Uccella N, Biophenolic components of olives. Food Res Int 33:475-485 (2000).

9 Visioli F, Poli A and Gall C, Antioxidant and other biological activities of phenols from olives and olive oil. Med Res Rev 22:65-75 (2002).

10 Tripoli E, Giammanco M, Tabacchi G, Di Majo DD, Giammanco S and La Guardi M, The phenolic compounds of olive oil: structure, biological activity and beneficial effects on human health. Nutr Res $\operatorname{Rev}$ 18:98-112 (2005).

11 Morello JR, Vuorela S, Romero MP, Motilva MJ and Heinonen M, Antioxidant activity of olive pulp and olive oil phenolic compounds of the Arbequina cultivar. J Agric Food Chem 53:2002-2008 (2005).

12 Bouaziz M, Chamkha M and Sayadi S, Comparative study on phenolic content and antioxidant activity during maturation of the olive cultivar Chemlali from Tunisia. J Agric Food Chem 52:5476-5481 (2004).

13 Rios JJ and Gutiérrez-Rosales F, Comparison of methods extracting phenolic compounds from lyophilised and fresh olive pulp. LWT - Food Sci Technol 43:1285-1288 (2010).

14 Morelló JR, Romero MP and Motilva MJ, Effect of the maturation process of the olive fruit on the phenolic fraction of drupes and oils from Arbequina, Farga, and Morrut cultivars. J Agric Food Chem 52:6002-6009 (2004).

15 Ocakoglu D, Tokatli F, Ozen B and Korel F, Distribution of simple phenols, phenolic acids and flavonoids in Turkish monovarietal extra virgin olive oils for two harvest years. Food Chem 113:401-410 (2009).

16 Alkan D, Tokatli F and Ozen B, Phenolic characterization and geographical classification of commercial extra virgin olive oils produced in Turkey. J Am Oil Chem Soc 89:261 -268 (2012).

17 Amiot MJ, Fleuriet A and Macheix JJ, Accumulation of oleuropein derivative during olive maturation. Phytochemistry 28:67-69 (1989).

18 Dağdelen A, Tümen G, Özcan MM and Dündar D, Phenolics profiles of olive fruits (Olea europaea L.) and oils from Ayval $l k$, Domat and Gemlik varieties at different ripening stages. Food Chem 136:41-45 (2013).

19 Menz G and Vriesekoop F, Physical and chemical changes during the maturation of Gordal Sevillana olives (Olea europaea L., cv. Gordal Sevillana). J Agric Food Chem 58:4934-4938 (2010). 